\title{
A near-infrared hyperspectral imaging system for quantitative monitoring of hemodynamics and metabolism on the exposed cortex of mice
}

Luca Giannoni, Frédéric Lange, Ilias Tachtsidis

Luca Giannoni, Frédéric Lange, Ilias Tachtsidis, "A near-infrared hyperspectral imaging system for quantitative monitoring of hemodynamics and metabolism on the exposed cortex of mice," Proc. SPIE 11074, Diffuse Optical Spectroscopy and Imaging VII, 1107413 (11 July 2019); doi: $10.1117 / 12.2526599$ 


\title{
A near-infrared hyperspectral imaging system for quantitative monitoring of hemodynamics and metabolism on the exposed cortex of mice
}

\author{
Luca Giannoni* $^{*}$, Frédéric Lange ${ }^{\mathrm{a}}$, Ilias Tachtsidis ${ }^{\mathrm{a}}$ \\ ${ }^{a}$ Department of Medical Physics and Biomedical Engineering, University College London, Malet \\ Place Engineering Building, Gower Street, London WC1E 6BT, UK
}

\begin{abstract}
A near-infrared (NIR) hyperspectral imaging (HSI) system has been developed to measure the hemodynamic (changes in concentration of oxyhemoglobin and deoxyhemoglobin) and the metabolic (changes in concentration of oxidised cytochrome-c-oxidase) responses in the exposed cortex of small animals. Using the extended spectral information of multiple wavelengths in the NIR range between 780 and $900 \mathrm{~nm}$ optimal differentiation between the optical signatures of the chromophores (hemoglobin and cytochrome-c-oxidase) can be achieved. The system, called hNIR, is composed of: (1) a high-frame rate, large-format scientific CMOS (sCMOS) camera for image acquisition and (2) a broadband source coupled with a Pellin-Broca prism mounted on a rotating motor for sequential, fast-rate illumination of the target at different spectral bands. The system characterisation highlights the capability of the setup to achieve high spatial resolution over a $\sim 1 \mathrm{x} 1 \mathrm{~mm}$ field of view (FOV). Hyperspectral data analysis also includes simulations using a Monte Carlo optical model of HSI, to estimate the average photon pathlength and improve image reconstruction and quantification. The hNIR system described here is an improvement over a previously tested commercial snapshot HSI solution both in terms of spatial resolution and signal-to-noise ratio (SNR). This setup will be used to monitor brain hemodynamic and metabolic changes in the exposed cortex of mice during systemic oxygenation changes.
\end{abstract}

Keywords: Hyperspectral imaging, near-infrared spectroscopy, diffuse optical imaging, brain metabolism, cytochromec-oxidase, brain oxygenation, brain hemodynamics

\section{INTRODUCTION}

HSI is an optical imaging technique involving the acquisition of 3D spatio-spectral datasets, called hypercubes, composed of multiple images at different wavelength bands. The application of HSI to in vivo brain imaging has demonstrated the potential to quantitatively monitor cerebral tissue oxygenation and hemodynamics in both small animals and humans, by detecting changes in reflected light intensity at numerous contiguous spectral bands, in the visible and NIR range [1]. This is typically done by measuring the variations in light absorption in the brain, due to changes in the relative concentrations of oxygenated $\left(\mathrm{HbO}_{2}\right)$ and deoxygenated $(\mathrm{HHb})$ hemoglobin. Additionally, the broad spectral information provided by HSI can be used to quantify in vivo cerebral metabolism, by targeting the relative changes in concentration of the oxidized form of cytochrome-c-oxidase (oxCCO) [1,2]. CCO is a key enzyme involved in more than $95 \%$ of the energy production of mitochondria in the neurons and it has a predominant absorption peak in the NIR range between 780 and $900 \mathrm{~nm}[2,3]$. Using a large number of wavelength bands over this spectral range can significantly improve differentiation between the optical signatures of hemoglobin and CCO [4]. We previously evaluated the potential of HSI for brain hemodynamic and metabolic monitoring using a commercial snapshot hyperspectral solution, targeting the exposed cerebral cortex of mice [5]. The results of the study stressed the importance of achieving high spatial resolution and SNR in the hypercubes. Therefore, a benchtop HSI system has been developed aiming to overcome the limitations of the commercial instrumentation and to demonstrate the feasibility of using this technology for in vivo imaging of the hemodynamic and metabolic states of the exposed cortex of small animals.

\section{METHODS AND MATERIALS}

The proposed NIR HSI system, name hNIR, is based on spectral scanning acquisition mode, where each spatial image is acquired sequentially for each different wavelength band, in order to create the hypercubes [1]. This method has the advantage of using the whole detecting area of the imaging camera, without need of multiplexing the spectral

Diffuse Optical Spectroscopy and Imaging VII, edited by Hamid Dehghani, Heidrun Wabnitz, Proc. of SPIE-OSA Vol. 11074, 1107413 · @ 2019 SPIE-OSA · CCC code: 1605-7422/19/\$21 · doi: 10.1117/12.2526599 
information, though at the cost of a reduced hypercube acquisition rate and of an increased sensitivity to motion artifact. A diagram of the system is depicted in Figure 1.

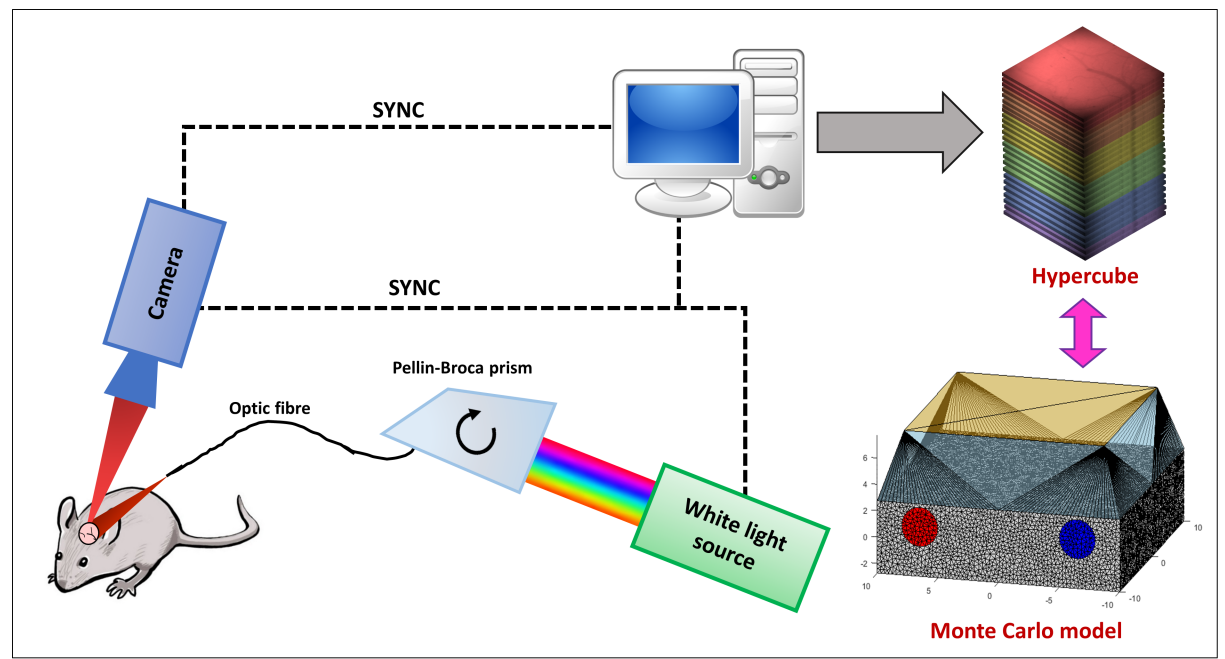

Figure 1. Diagram of the HSI system, including illumination and imaging side. The setup can be integrated with a Monte Carlo optical model to aid image reconstruction and estimation of quantities, such as average photon pathlength.

\subsection{Instrumentation}

The hNIR is composed of a broadband white light source (Oriel 66088) that is coupled with a Pellin-Broca mounted on a rotating motor (PI U-651.04) for sequential illumination of the target at different wavelengths between 780 and $900 \mathrm{~nm}$ at variable spectral sampling $(5-20 \mathrm{~nm})$. The motor provides an average wavelength band switching rate of $20 \mathrm{~ms}$. A picture of the illumination side of the setup is shown in Figure 2a. Image acquisition is performed using a sCMOS camera (Andor Zyla 5.5 USB 3) with 5.5-megapixel format and $6.5-\mu \mathrm{m}$ pixel size. The camera can run at maximum frame rate of $400 \mathrm{fps}$ (at 512x512 pixel binning). The camera is coupled to an optical assembly including: (1) a $15 \mathrm{x}$ reflecting objective (Thorlabs LMM-15X-P01) covering visible and IR range (450-2000 nm) and (2) an infinitycorrected relay lens tube (Thorlabs TTL200-B). Figure $2 b$ depicts the imaging side of the system. This provides a FOV of $1.1 \times 0.9 \mathrm{~mm}$ with average spatial resolution of $228 \mathrm{lp} / \mathrm{mm}$, as shown in Figure 2c and $2 \mathrm{~d}$.
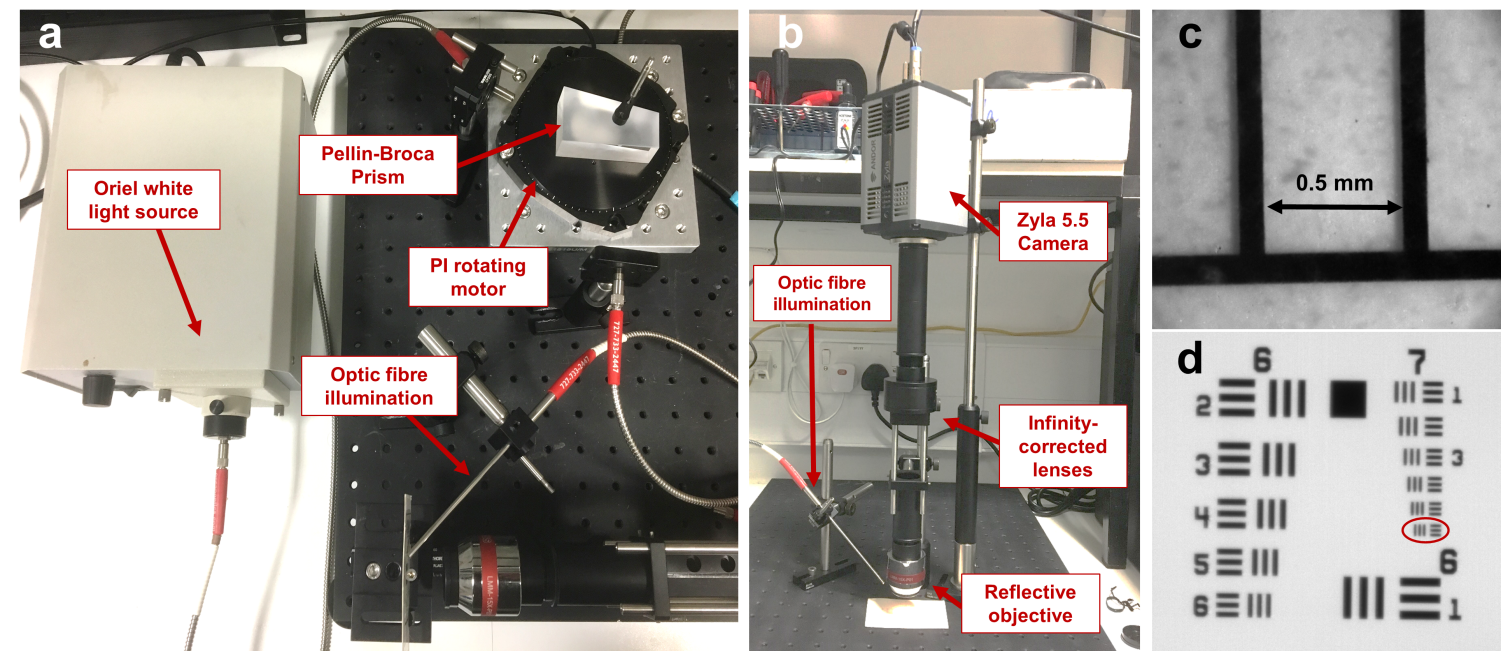

Figure 2. a) Picture of the illumination side of the HSI system; b) Picture of the imaging side of the HSI system; c) Image of a calibrated target taken with the HSI system, showing a 1.1x0.9 mm FOV; d) Image of a high resolution sample target (USAF 1951) taken with the HSI system. The smallest resolvable element (in red) was identified as Group 7, Element 6, corresponding to a spatial resolution of $228 \mathrm{lp} / \mathrm{mm}$. 


\subsection{Data processing}

The hypercube acquired with the HSI system are pre-processed and time-averaged to maximise SNR before performing spatial quantification of the relative changes in concentration of the targeted chromophores, i.e. $\Delta\left[\mathrm{HbO}_{2}\right], \Delta[\mathrm{HHb}]$ and $\Delta[\mathrm{oxCCO}]$ for $\mathrm{HbO}_{2}, \mathrm{HHb}$ and oxCCO respectively. These are obtained by applying the modified Beer-Lambert's law (MBLL) for each pixel of the hypercubes [6], in order to obtain hemodynamic and metabolic maps of the changes in the exposed cortex during different experimental conditions. Estimation of the average photon pathlength is achieved via simulations using a Monte Carlo optical system based on Meshed-Monte Carlo (MMC) [7]. This model has been developed to replicate the geometry and optical properties of the target cerebral tissue, as well as the HSI configuration used in the experiments.

\section{CONCLUSIONS}

The characterization of the hNIR system has shown important improvement and advantages over the commercial snapshot solution previously investigated [5]. In particular, the 5.5-megapixel format of the hNIR provides higher spatial resolution than the 1-megapixel detection area of the commercial system, with smaller FOV (1x1 mm versus $2 \times 2 \mathrm{~cm}$ of the snapshot solution) and higher SNR. Although the spectral scanning method used by the hNIR is technically slower than the snapshot acquisition, the high wavelength switching rate allows the benchtop system to achieve a hypercube rate comparable to the commercial solution one (at the cost of a lower number of imaged spectral bands). Finally, the hNIR has also a considerably lower cost than the commercial snapshot solution.

The hNIR system will be used on the exposed cortex of mice during different oxygenation stimuli (normoxia, hypoxia and hyperoxia), by changing the fraction of inspired oxygen $\left(\mathrm{FiO}_{2}\right)$. The quantitative images of the exposed cortex that can be obtained could provide significant information regarding the hemodynamic and metabolic states of the brain, enabling a deeper and more exhaustive understanding of cerebral physiology and of the optical origin of the brain signals that are currently measured non-invasively via near-infrared spectroscopy (NIRS) and diffuse optical imaging (DOI) [2].

\section{ACKNOWLEDGEMENTS}

LG is supported by the European Union's Horizon 2020 research and innovation programme under the Marie Sklodowska-Curie grant agreement No 675332. FL and IT are supported by the Wellcome Trust (104580/Z/14/Z).

\section{REFERENCES}

[1] Giannoni, L., Lange, F. and Tachtsidis, I., "Hyperspectral imaging solutions for brain tissue metabolic and hemodynamic monitoring: past, current and future developments," J. Opt 20(4), 044009 (2018).

[2] Bale, G., Elwell, C. E. and Tachtsidis, I. "From Jöbsis to the present day: a review of clinical near-infrared spectroscopy measurements of cerebral cytochrome-c-oxidase," J. Biomed. Opt. 21(9), 091307 (2016).

[3] Mason, M. G., Nicholls, P. and Cooper, C. "Re-evaluation of the near infrared spectra of mitochondrial cytochrome c oxidase: implications for non invasive in vivo monitoring of tissues," Biochim. Biophys. Acta 1837(11), 1882-1891 (2016).

[4] Arifler, D., Xhu, T., Madaan, S. and Tachtsidis, I., "Optimal wavelengths combinations for near-infrared spectroscopic monitoring of changes in brain tissue hemoglobin and cytochrome c oxidase, " Biomed. Opt. Exp. 6(3), 933-947 (2015).

[5] Giannoni, L., Lange, F. and Tachtsidis, I., "Hyperspectral imaging of the hemodynamic and metabolic states of the exposed cortex: investigating a commercial snapshot solution, " Adv. Exp. Med. Biol. 1072, 13-20 (2018).

[6] Delpy, D. T., Cope, M., van der Zee, P., Wray, S. and Wyatt, J., " Estimation of optical pathlength through tissue from direct time of flight measurements, " Phys. Med. Biol. 33(12), 1433-1442 (1988).

[7] Fang, Q., "Mesh-based Monte Carlo method using fast ray-tracing in Plücker coordinates, " Biomed. Opt. Exp. 1(1), 165-175 (2010). 